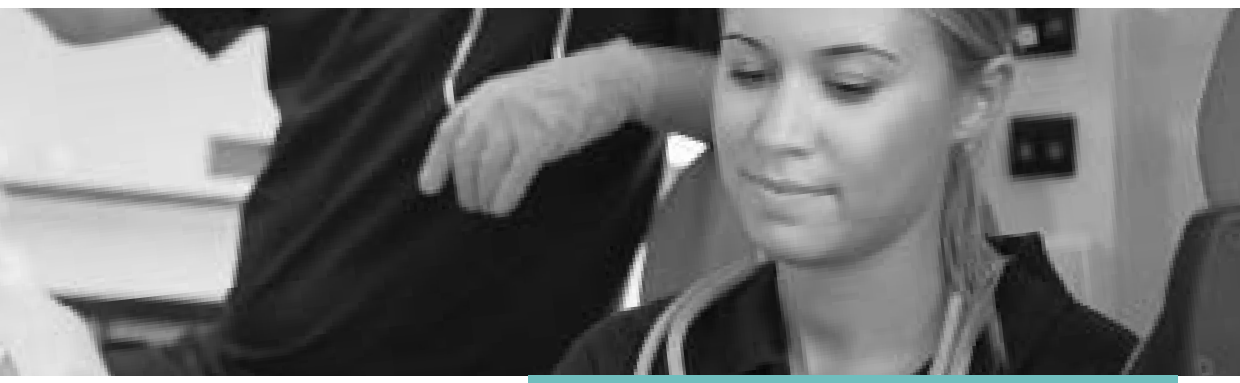

\title{
AUSTRALASIAN JOURNAL OF PARAMEDICINE
}

Fostering a research culture in paramedicine: Selected proceedings from the 2011-2013 Paramedic Research Forum at Auckland University of Technology

\section{Bronwyn Tunnage}

Auckland University of Technology, New Zealand 


\title{
Conference abstracts
}

\section{Fostering a research culture in paramedicine: Selected}

\section{proceedings from the 2011-2013 Paramedic Research Forum at Auckland University of Technology}

\author{
Bronwyn Tunnage MSc ${ }^{1}$
}

\author{
Affiliation: \\ ${ }^{1}$ Auckland University of Technology, New Zealand \\ Corresponding Author: Bronwyn Tunnage, bronwyn.tunnage@aut.ac.nz
}

Pre-hospital emergency care has undergone a paradigm change over the past 40 years. Paramedics have transformed from stretcher-bearers, whose primary role was transporting the patient, to medical specialists $(1,2)$ providing care in the community and transitioning the patient to the appropriate primary, secondary or tertiary healthcare facility. However, research in this emergent profession has not kept pace with the rapidly changing scope of practice (3). The increasing complexity of the paramedic role and the requirement for advanced clinical and decision-making skills has generated a shift from vocational training to entry through tertiary education, in alignment with other healthcare professions (4). Curricula within paramedic undergraduate programs in Australia and New Zealand are shaped by the independent and external assessment and accreditation processes of the Council of Ambulance Authorities (4). The need for research literacy and activity is expressly acknowledged by the Paramedic Professional Competency Standards in the requirements for an understanding of the research process and for practice to be evidence-based (5). Providers of paramedic education have a responsibility to foster a strong research culture to support evidence-based practice within paramedicine.

The delivery of clinical care that is informed by research findings demonstrating improved patient outcomes should be the foundation of healthcare delivery at all stages of the patient journey (6). However, the widespread adoption of evidence-based practice in paramedicine faces several challenges. Utilisation of research findings in emergency medical services (EMS) depends on paramedics having sufficient literacy to locate and evaluate relevant studies. While accreditation provides assurance of research literacy among graduates of approved programs, vocational training has maintained a focus on operational competencies, creating a workforce with mixed abilities to apply research knowledge to practice. A further obstacle to bridging the evidencepractice gap is the scarcity of publications in the field of paramedicine. This is exacerbated by insufficient research capacity and a relative absence of advanced scholarship in comparison to other healthcare disciplines. One option, to generalise findings from other healthcare professions and practice settings, is not necessarily appropriate. Paramedic practice is arguably defined by the uncontrolled environment with constrained personnel and resources that other healthcare professionals do not face (7). Similarly, the validity of generalising international results to the local EMS context, where care is provided to a culturally different population, in a geographically dissimilar environment, within a distinct healthcare system, is undetermined. Paramedics need to participate in research in the location where they practise.

Promoting a paramedic-led research culture within EMS is the guiding objective of the Paramedic Research Forum. The forum is convened annually to disseminate findings from the individual research projects completed by students in their final year of the Bachelor of Health Science in Paramedicine at Auckland University of Technology. The student project is the culmination of a stepped learning experience embedded throughout the degree that progresses students from novices to informed consumers and finally producers of research. Students investigate a topic arising from reflections on their clinical experience and produce three outputs: a written report in the format of a journal paper, plus oral and poster presentations that are submitted in the conference setting. The forum attracts delegates from across the sector including operational, educational and managerial staff from EMS provider St John (New Zealand) academic staff from other paramedic programs and the Defence Health School New Zealand, and from allied industries and professional representative bodies. This creates a nexus across the broader sector, and particularly between EMS providers and academia, which endorses and champions evidence-based practice. For the students, the experience of successfully completing an individual study based on the paramedic practice setting demystifies the research process, generating an enthusiasm that has led to postgraduate study and publication (8). 
Selected proceedings from the 2011-2013 Paramedic Research Forums are presented here in poster format. These presentations all received commendations and they demonstrate the breadth of topics selected, encompassing both clinical and operational issues. Through the promotion of a vigorous research culture within the nascent profession, the Paramedic Research Forum contributes to the adoption of evidence-based practice in paramedicine.

\section{References}

1. Simpson AT. Transporting Lazarus: physicians, the state, and the creation of the modern paramedic and ambulance, 1955-73. J Hist Med Allied Sci 2013;68(2):163-97.

2. Wallis J, Boyle M. From stretcher bearer to 'Paramedic'. Australasian Journal of Paramedicine 2014;11(3).

3. Archer F, Woollard M, Snooks H, et al. What are the highest priorities for research in pre-hospital care? Results of a review and Delphi consultation exercise. Australasian Journal of Paramedicine 2008;6(4).

4. O'Brien K, Moore A, Dawson DA, Hartley PR. An Australian story: paramedic education and practice in transition.

Australasian Journal of Paramedicine 2014;11(3).
5. Council of Ambulance Authorities. Paramedic Professional Competency Standards. Version 2.2, 2013. Available at: www.caa.net.au/attachments/article/91/Paramedic $\% 20$ Professional\%20Competency $\% 20$ Standards $\% 20 \mathrm{~V} 2.2 \% 20$ February $\% 202013 \% 20 \% 20$ PEPAS.pdf

6. Donoghue J. Research for emergency care. In: Curtis K, Ramsden C, Lord B, editors. Emergency and Trauma Care for Nurses and Paramedics. Australia: Mosby; 2011.

7. Campeau A. Introduction to the 'space-control theory of paramedic scene management'. Emerg Med J 2009;26(3):213-6.

8. Wallen R, Tunnage B, Wells S. The 12 lead ECG in the EMS setting: how electrode placement and paramedic gender are experienced by women. Emerg Med J 3 July 2013. [Epub ahead of print]. 



\section{The Effect of Stimulus on Intracranial Pressure in Traumatic Brain Injury}

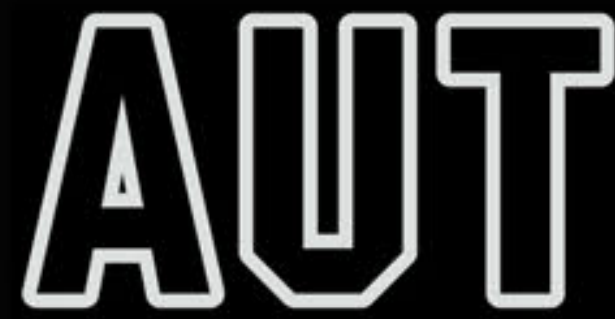

U N I V E R I T Y

\section{Curtis Akitt}

\section{Introduction}

Traumatic brain injuries (TBI) account for half of all deaths associated with trauma and are the leading cause of death for under 40 year olds $(1,2)$.

New Zealand incidences of TBl's have been significantly increasing, with an estimated 226.9 people per 100,000 in $1997-8$ rising to 349.2 in

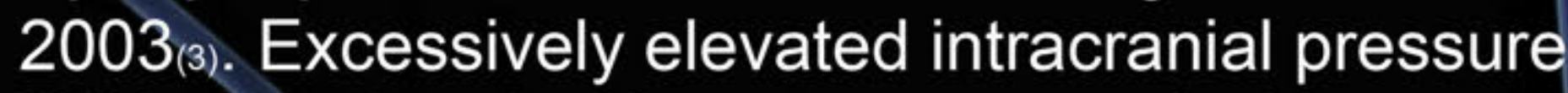
(ICP) is correlated with poor outcomes in this group of patients and is present in over half of all TBI deaths $(4)$.

External stimulus has been said to increase ICP in TBI patients ${ }_{(5,6,7)}$, potentially contributing to increased mortality. This papers focus was to look at current literature and research on the subject, and if stimulus does increase ICP.

\section{Results}

Of the 17 relevant literature items $82 \%$ stated that noxious and other stimuli increase ICP in TBI patients, with $11 \%$ not stating specifically it does increase ICP, but acknowledging that unconscious patients can respond to stimuli, and $6 \%$ suggesting that thoracic stimuli, decreases ICP. One recent

study that looked at $252-17$ year olds with moderate to severe TBI's found that log rolls and endotracheal suctioning increased ICP by $8.6-8.7$ $\mathrm{mmHg}$ on average ${ }_{(5)}$. During this, $70 \%$ of patient's exceeded $20 \mathrm{mmHg}$, and $28 \%$ exceeded $30 \mathrm{mmHg}$ which/is clinically significant when the threshold for treatment is $20-25 \mathrm{mmHg}(5)$.

\section{Method}

A systematic literature review using keywords was conducted using a number of prominent medical databases including Medline and CINAHL. Current research was used from 2001 onwards and returned 17 relevant

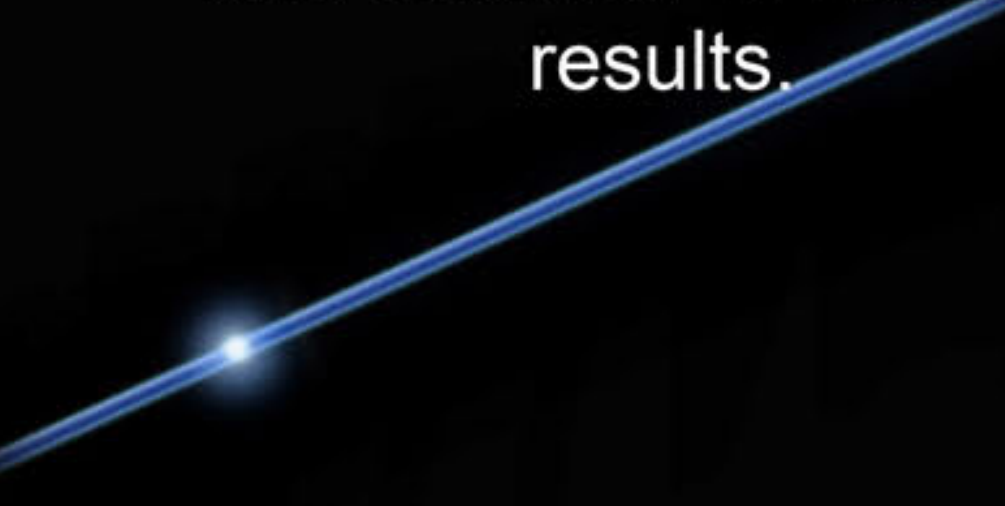

- Noxious, aggressive and invasive types of stimulus have been shown to increase ICP in TBI patient's and is a widely accepted concept, however there is limited current research $(5,6,7)$.

- Clinically significant spikes in ICP have been theorised to have the ability to facilitate the transition from compensated to decompensated brain injury states in TBI patients (8) which could lead to increased mortality.

- Analgesia and sedatives are currently used to limit ICP spikes in hospital environments (9,10) and research has suggested that this approach could be utilised in pre-hospital environments safely with accurate support and guidelines (11).

1. Heegaard, W., \& Biros, M. (2007). Traumatic brain injury. Emergency medicine clinics of North America, 25(3), 655-678. doi:10.1016/j.emc.2007.07.001

outcomes from tra Cooper, D. J., Finfer, S. R., Venkatesh, B., Jones, D., Higgins, A., ... Higlett, T. (2008). Epidemiology and 12-month doi:10.1097/TA.0b013e3180340e77

3, Barker-Collo, S. L., Wilde, N. J., \& Feigin, V. L. (2009). Trends in Head Injury Incidence in New Zealand: A Hospital-Based Study from 1997/1998 to 2003/2004. Neuroepidemiology, 32(1). 32-32-39, doi:10.1159/000170090
7, Reddy, L. C. S. (2006). Heads up on cerebral bleeds. Nursing, 36(5), 4. Retrieved from

8, Fan, J. Y., Kirkness, C., Vicini, P., Burr, R., \& Mitchell, P. (2010). An approach to determining intracranial pressure variability capable of predicting decreased intracranial adaptive capacity in patients with traumatic brain injury. Biological research for nursing, 11(4), 317. doi: 10.1177/1099800409349164

9, Ladanyi, S., \& Ellott, D. (2008). Traumatic brain injury: An integrated clinical case presentation and literature review:: Part

10, Lescot, T., Abdennour, L., Boch, A. L., \& Puybasset, L. (2008). Treatment of intracranial hypertension. Current Opinion in 11. Pediatric Critical Care Medicine. (2003) Chapter 92 Use of 
Introduction: A limitation of pre-hospital patient assessment is the identification of haemodynamic instability due to physiological parameters, of which assessment is based, remaining stable despite a critical underlying pathology. Lactate levels are hypothesised to be a more sensitive parameter in the true evaluation of a patient's haemodynamic status than conventional vital signs alone (Jansen et al., 2008).

Methodology: A literature review was undertaken, as illustrated in figure

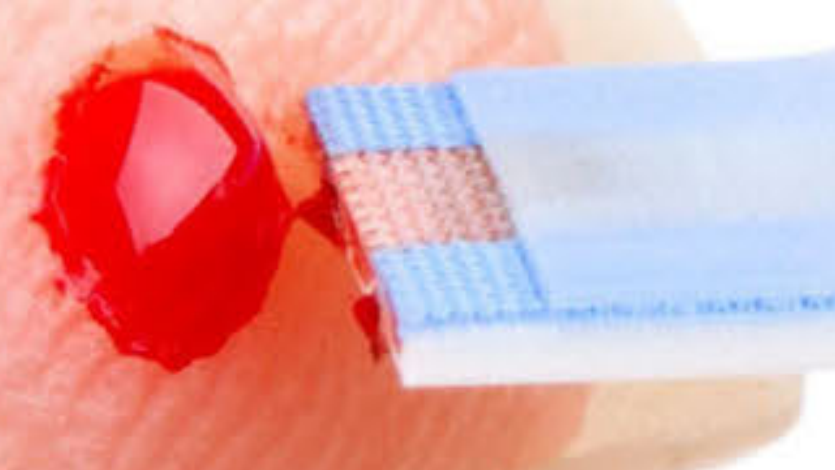

Figure 1: Search strategy utilised in literature review
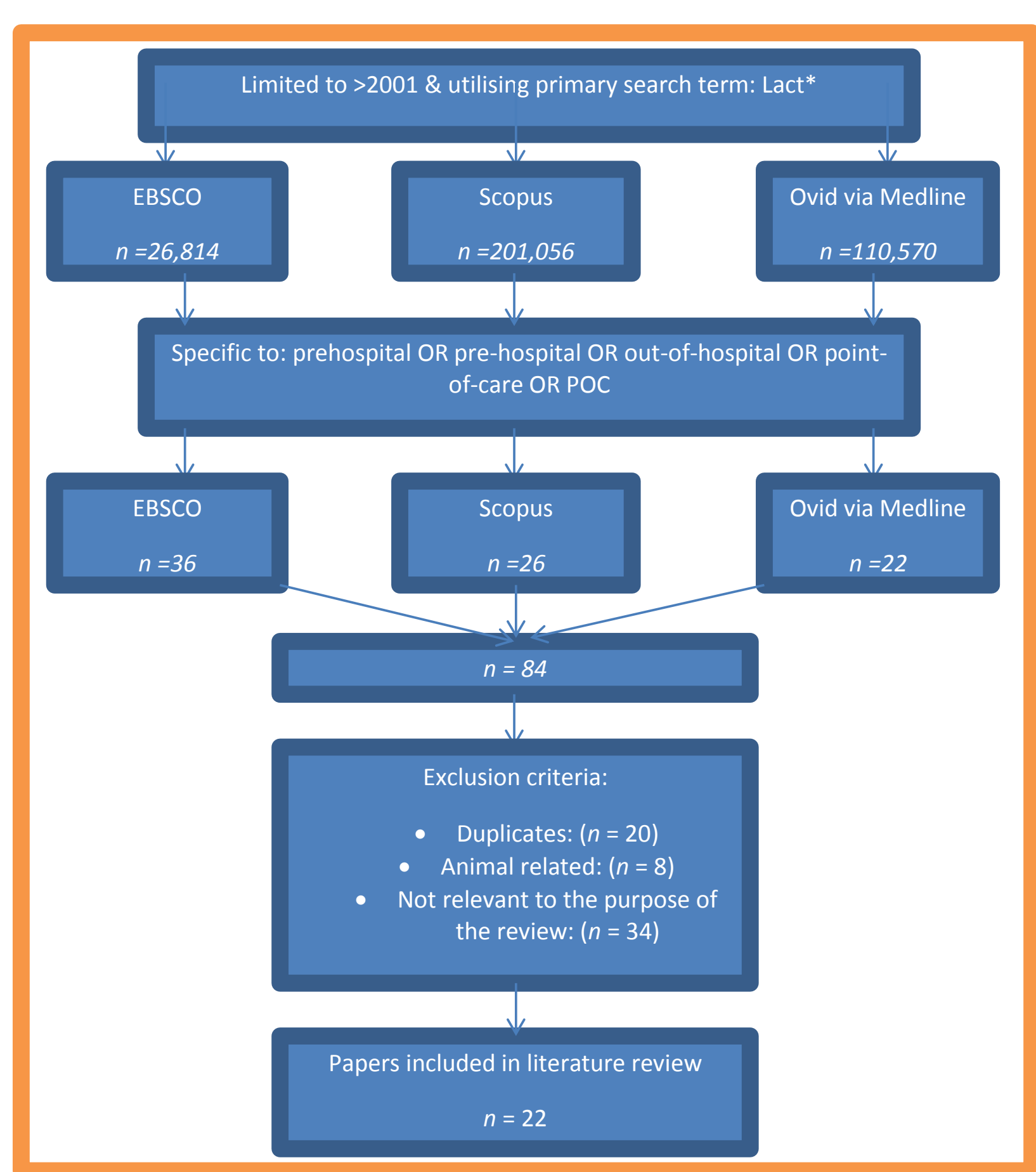

Results: Four key themes were identified from the literature:

1. Accuracy of POC lactate versus laboratory values are clinically acceptable

2. Lactate provides prognostic benefit which enhances risk stratification and therefore triage

3. Lactate aids in the identification of occult presentations of shock

4. Lactate is useful as a sole parameter to initiate goal directed therapy, especially for septic shock

Limitations: The primary limitation of this study is the lack of research into the efficacy of POC lactate in the pre-hospital setting.

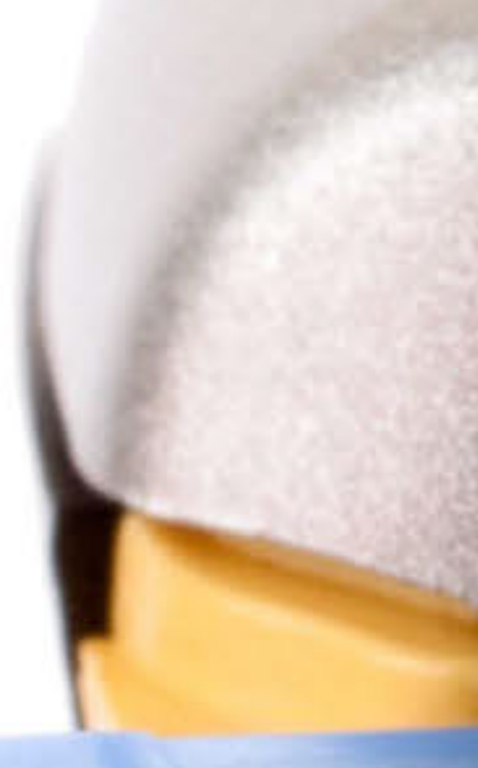

\begin{abstract}
The evidence supporting the efficacy of POC lactate, although limited, suggests significant clinical benefit from its use. Consideration should therefore be given to its implementation in the New Zealand pre-hospital sector, with further in-depth research being a fundamental
\end{abstract} pre-requisite before its adoption into practice.

Lactate is potentially a more sensitive marker of haemodynamic instability than vital signs alone

References: (1): Jansen, T. C., Bommel, J. V., Mulder, P. G., Rommes, J. H., Schieveld, S. J., \& Bakker, J. (2008). The prognostic value of blood lactate levels relative to that of vital signs in the pre-hospital setting: a pilot study. Journal 


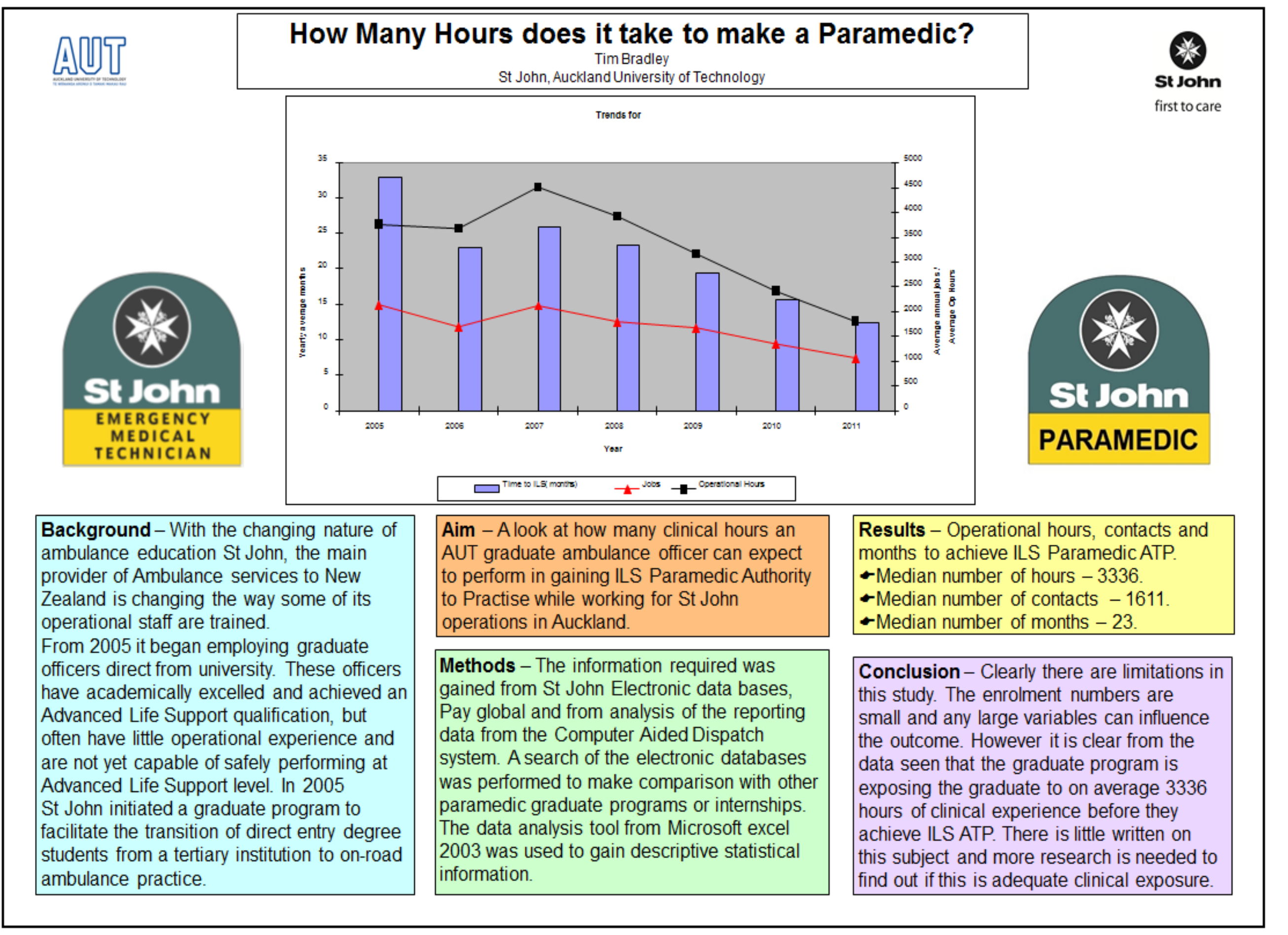




\section{Is Critical Incident Debriefing of Emerge
Beneficial or Harmful?}

\section{Introduction}

Emergency personnel are exposed to traumatic events almost on a daily basis. These critical incidents have the potential to cause serious physiological harm to individuals, therefore as an industry, paramedicine must look at effective ways to minimise harm and promote support to individuals and teams. By reviewing available studies we can gain a better understanding of the risks and benefits of Critical Incident Debriefing along with other alternative strategies that may benefit staff exposed to traumatic events.

\section{Method}

A systematic electronic database search was conducted in EBSCO. The search terms used included critical incident, debrief*, crisis, stress, benefit ${ }^{*}$, harm ${ }^{*}$, trauma and emergency with inclusion criteria of English and humans and a search date of 2005-2013. All titles and available abstracts were read to identify papers potentially addressing the research question (see table 1). Full texts of the relevant articles were then obtained and reviewed. The rationale behind the exclusions was to concentrate on the impact of debriefing on emergency workers as secondary victims who have a higher exposure to critical incidents.

\section{Results/Discussion}

Two Emergency Department studies (table 2) showed that the vast majority of emergency workers in the study felt that debriefing a critical incident was important

In a study of ED doctors and nurses, respondents perceived debriefings as an opportunity to:

Provide staff with emotional or psychological support Help staff improve or review clinical practice Foster team spirit

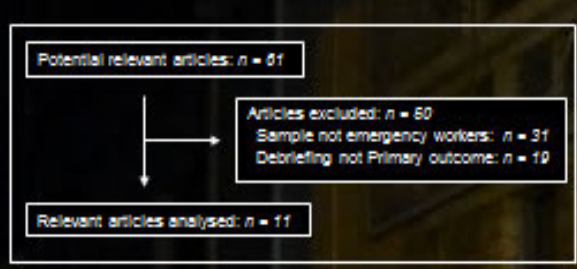

Table 1. Summary of selection process

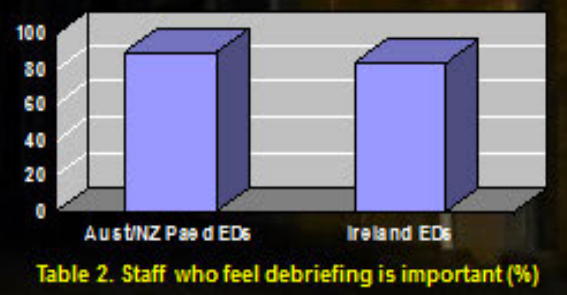

\section{Conclusions}

Emergency workers have a clear preference to being able to debrief after critical incidents. Potential harm can be mitigated by formal debriefs being taken by trained, credible people who are independent from the actual incident, where debriefs are not held within $24 \mathrm{hrs}$, and when they are not compulsory to attend.

Recommendations for th

- Undertake research within New Zealand on the effects of critical incidents

- Develop a robust proces and manage fent of criticalincider

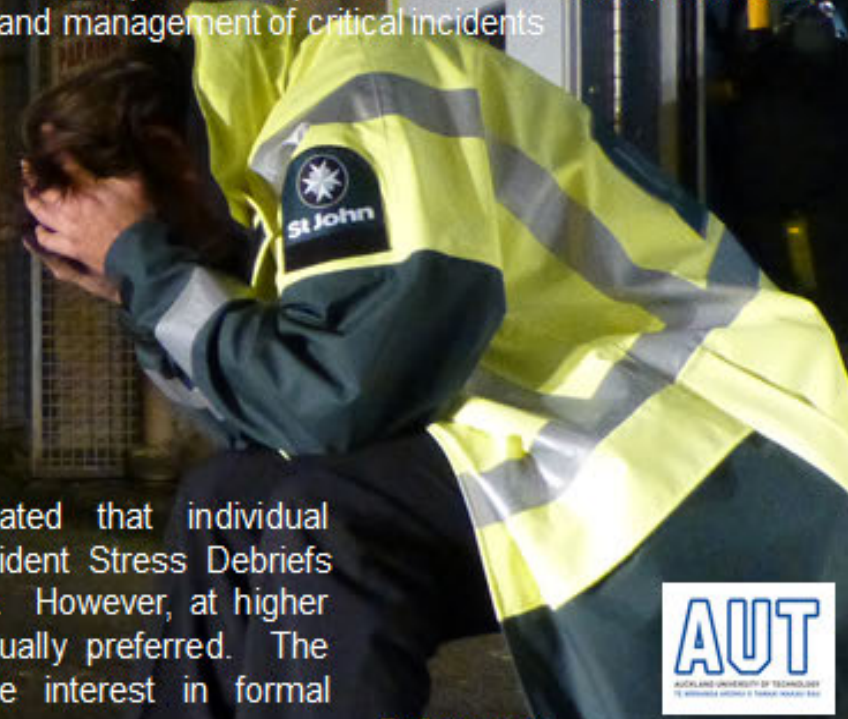

A Canadian study of Firefighters indicated that individual debriefings were preferred over Critical Incident Stress Debriefs (CISD) for low to moderate severity events. However, at higher severity both individual and CISD were equally preferred. The more severe the incident, the higher the interest in formal intervention.

A Canadian EMT study highlighted the benefit of supportive supervisors in their acknowledgement of an incident, expression of concern about crew well-being, a willingness to listen, their offer of material help, and the valuing of the paramedic's efforts.

Debriefing was considered to be potentially harmful if:

Debriefing is offered too soon ( $<24 \mathrm{hrs})$

- Attendance is mandatory

Debrief is rushed Taken by untrained personnel

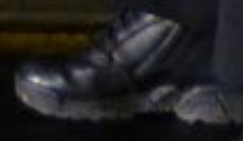

Organisations need to anticipate the effects of traumatic exposure and have strategies to deal with the effects in the workplace.

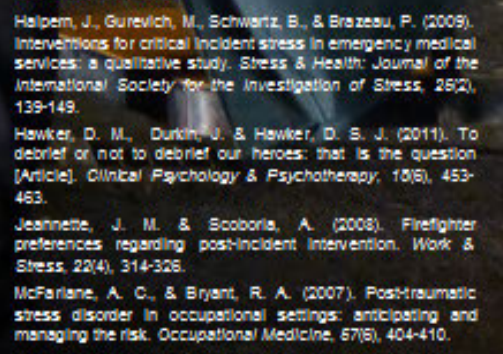

References 


\section{Factors that influence staff retention in an ambulance setting}

By Rebekah Judd

\section{Introduction \\ The aim of this literature review is to help positively identify what may influence ambulance staff to stay with their employer, without costing much money, potentially saving organisations money, time and experience.}

\section{Methods:}

Articles that researched staff retention and turnover factors within the health industry were considered, as were improving retention, healthier workplaces,

burnout, fatigue, cynicism, engagement, and leadership Searched: Wellbeing staff retention, medical, tumover, paramed ${ }^{*}$, and healthcare

Refined keywords included: Healthy workplaces, burnout, fatigue, cynicism, engagement, and leadership

\section{Resultse}

The search was narrowed down from an initial number of 217 articles, to 15 . This was done using the keywords of: Burnout, job satisfaction, staff wellbeing, and leadership; along with Paramedic, healthcare industry.

Limited to 15 relevant articles.

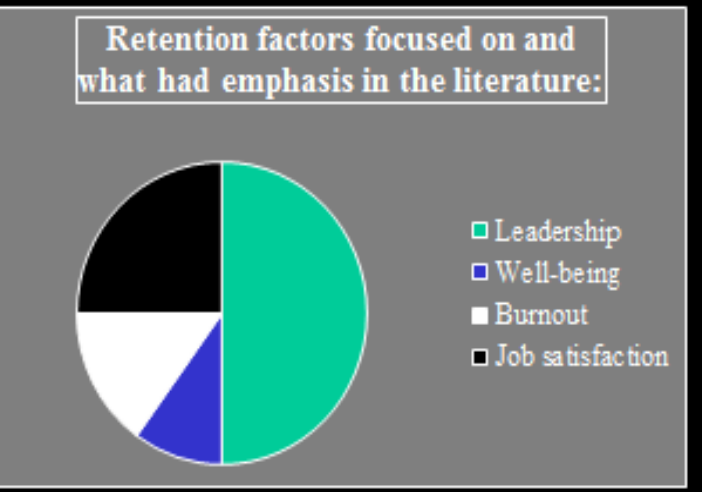

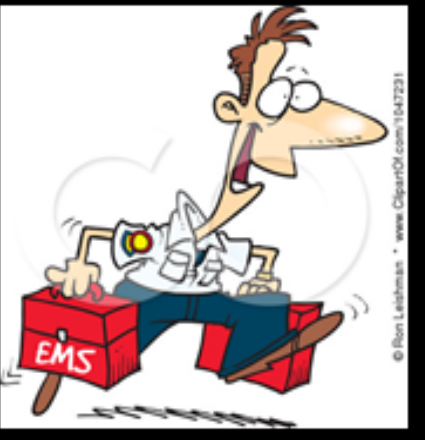
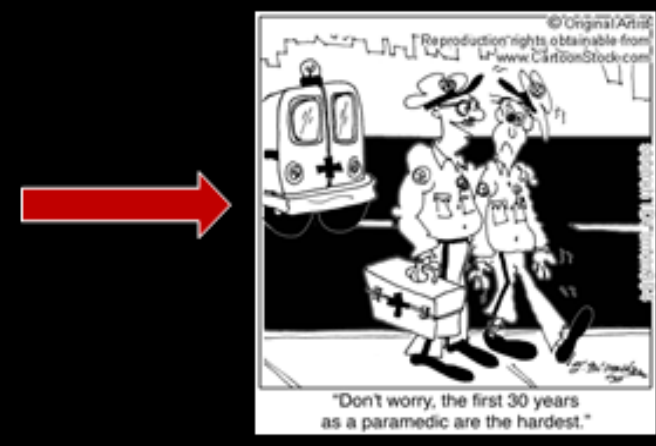

\section{Discussiont}

- Exhaustion + Cynicism + Inefficacy $=$ BURNOUT

- Workload for St John ambulance service in Auckland has increased, causing staff to become fatigued, disillusioned, cynical, uncivil.

- Ambulance staffare confronted by the rav emotions of patients and their families, sometimes by violence, by sadness beyond "normal" occupations imaginings; and also the day in day out grind of having an intense involvement with people. This may affect personal lives and general well-being

There is seen to be little opportunity to advance in the industry, and staff may feel their skills are not being utilised.

All these factors lead to job dissatisfaction, and are all major turnover factors - Leadership: Distrust in leadership, managers and organisations may affect wellbeing, causing greater stress, which may lead to job dissatisfaction, which may lead to burnout

- Staff often don't feel valued or thanked.

- Staff feel they don't visualise their leaders and feel they are not involved with any organisational decisions.

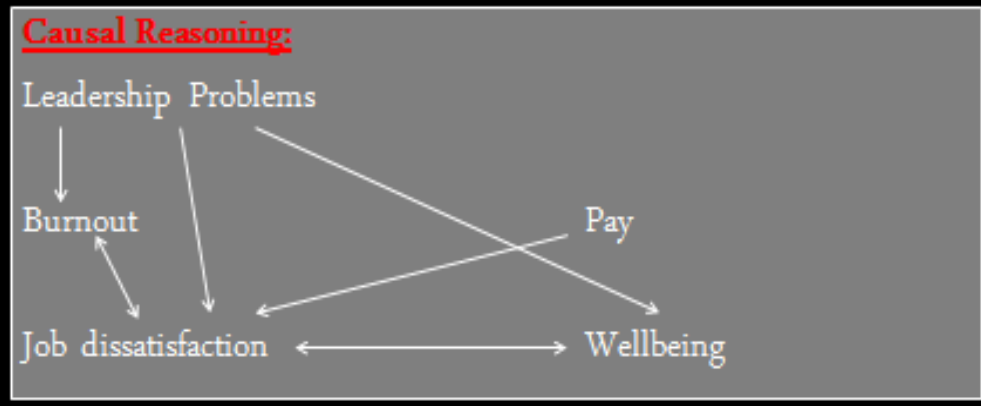

Conclusions:

- Bumout, job dissatisfaction, poorwellbeing, and poor leadership are well-known, well-studied retention factors.

Some implementations that may increase staff retention are:

A new, flexible, roster structure, where staff choose their roster may decrease burnout.

Empowerment and education will increase job satisfaction.

Increased awareness of well-being factors will benefit staff, such as education about decreasing stress and fatigue levels.

- Incentives to do well may include educational opportunities, or bonus shifts off after certain performance indicators are met.

Changes must be made to increase staff organizational commitment and trust

- Leadership has the most important to part to play in implementing positive changes that may entice staff retention: good communication with staff, and visibility, along with valuing staff and empowering them; may lead to a decrease in the other negative factors.

- Leading to greater retention increased job satisfaction, happier staff and a decreased intention to leave.

References:

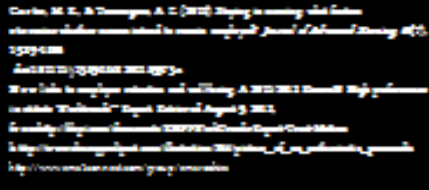




\section{GLUCAGON Use for Beta-Blocker \& Calcium Channel Blocker Overdose}

Introduction: Overdose from cardiovascular drugs is associated with significant morbidity and mortality. Death from cardiovascular drugs is the $5^{\text {th }}$ leading cause of poisoning according to a 2004 report by the Toxic Exposure Surveillance System. The wide availability of these prescription drugs makes them problematic in both cases of accidental and intentional overdose. Complications of $\mathrm{BB}$ and $\mathrm{CCB}$ overdose can be life-threatening and include symptomatic bradycardia, hypotension and altered levels of consciousness. In most cases we treat what we see which would usually involve Atropine, IV Fluids and Adrenaline, however in the case of $\mathrm{BB}$ and $\mathrm{CCB}$ toxicity these interventions are relatively ineffective due to the blockade of the sympathetic adrenergic receptors of cardiac muscle cells.

The first reported case of using glucagon for BB overdose was in 1973 and proved to have a positive effect even though the patient later died from urosepsis. Following this report the research and use of glucagon has increased with many case reviews reporting a significant increase in heart rate and blood pressure within 3-5 minutes after the administration of intravenous glucagon. The first reported case of glucagon relieving CCB induced hypotension was in 1993, although many are sceptical of its effectiveness due to fewer isolated reports of its ingestion.

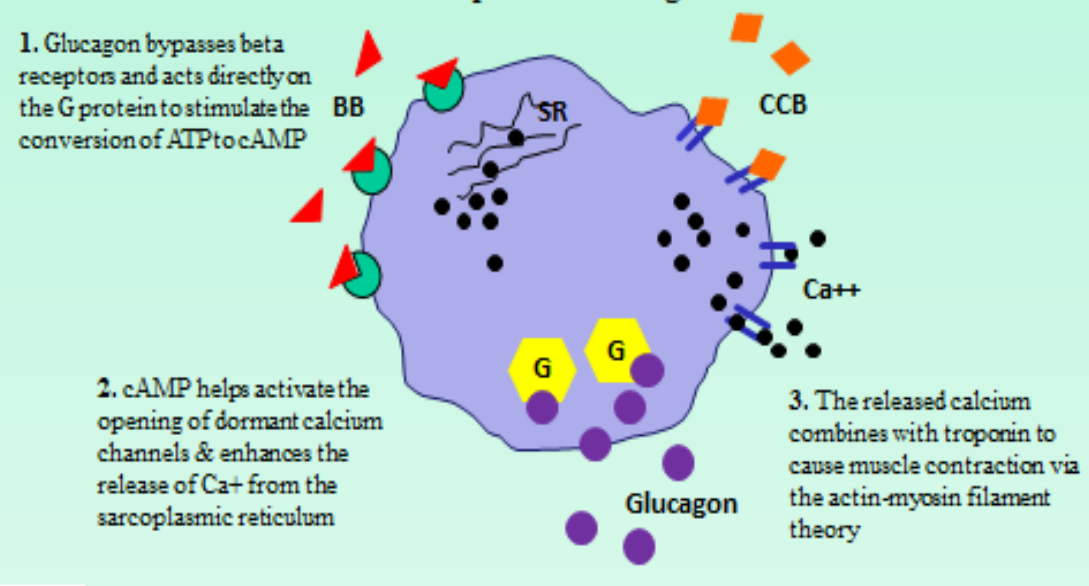

Te Paea KINGI

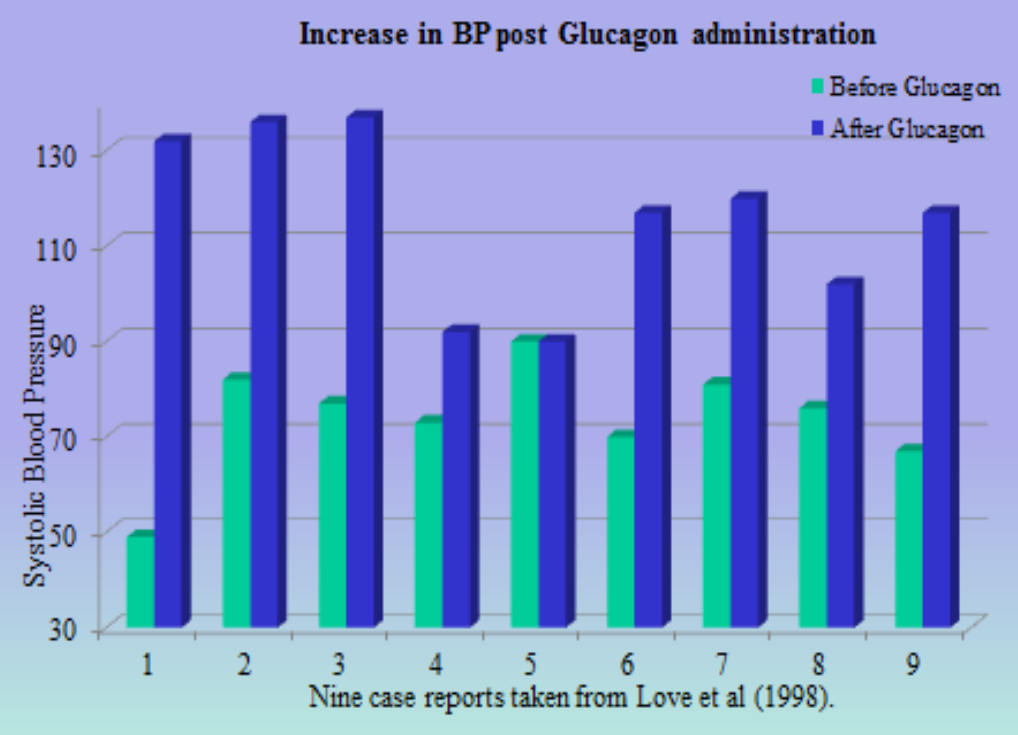

Method: This systematic review was conducted using an exclusion method from both sciencedirect and EBSCO databases. Search terms included "betablocker', 'calcium channel blocker', 'glucagon', 'overdose' OR 'toxicity'. A total of 49 articles were narrowed down further to 15 after excluding irrelevant research such as animal studies, repeated articles and letters to the editor Articles that required purchasing were also discarded. Due to the fact that there have been no controlled human trials, results are based on case reports.

Results: 13 of the 15 articles reported increases in blood pressure, heart rate and GCS following the initial bolus of between $1-10 \mathrm{mg}$ glucagon IV. An $3 \mathrm{mg} / \mathrm{hr}$ infusion of glucagon over the next 10-24hrs was given in conjunction with either norepinephrine or isoproterenol. In 2 of the case reports involving isolated calcium channel blocker overdose, glucagon failed to have any affect on blood pressure. There are no published reports of glucagon being used pre-hospitally in New Zealand for the management of BB and CCB toxicity, although it has been used on the ambulance and Westpac chopper under the authorisation of the St John medical director. It would be beneficial in the future for all use of glucagon to be reported and published as to promote it's efficacy in the treatment of symptoms associated with $\mathrm{BB}$ and $\mathrm{CCB}$ overdose. Evidence of it's effectiveness would facilitate it's addition into the ambulance national standards clinical guidelines. 


\section{Best practice in pre-hospital opiate management for paediatric pain}

Kate Livingston

\section{Introduction}

Paediatrics pose a considerable issue for pain management due to a number of barriers that hinder their assessment and management. This leaves some pain undiagnosed and untreated which can leave lasting effects. Overcoming these barriers is imperative to ensure paediatrics receive appropriate pain relief.

\section{Methods}

A systematic review of scholarly literature was conducted using Scopus, EBSCO and Cochrane databases. Included studies were limited to the last 10 years, peer-reviewed, full text, English language and pre-hospital studies. The remaining studies were then evaluated using the exclusion and inclusion criteria in order to find relevant and credible sources. To be utilized in this systematic review, studies had to be based on the use of opiate analgesia in paediatrics in the pre-hospital environment. Two prospective studies and four retrospective studies were utilised in this systematic review.

\section{Results}

All of the studies evaluated the use of opiate analgesia in paediatrics in the pre-hospital environment. The key findings in five of the articles concluded that age has a significant impact on the likelihood of receiving opiate analgesia. The majority of studies showed a low paediatric opiate administration rate with under 5 year olds receiving no pain relief in two of the studies.

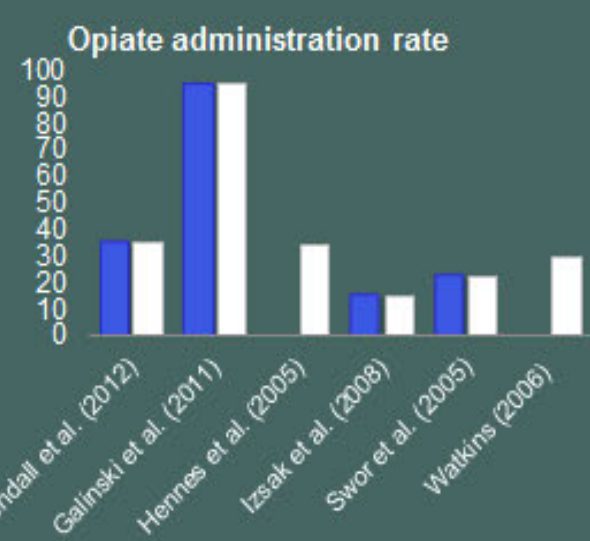

\section{Discussion and Conclusion}

Research surrounding pre-hospital opiate analgesia in paediatrics is scarce and is generally not of high quality. Despite this, age has emerged to be a significant predictor in the probability of receiving opiate analgesia. The factors influencing these judgements are uncertain however a consensus has been made within the literature that paediatrics are less likely to receive opiates. In conclusion, identifying the use of opiates in the pre-hospital environment provides opportunities to improve pain management practises. There is no one particular answer as to why paramedics use opiate analgesia less in children. However, the barriers identified can be minimized through the use of IN fentanyl, the improvement of assessing paediatric pain and further training in paediatric IV access. The development of health professionals in this area is likely to increase the number of paediatrics receiving analgesia.

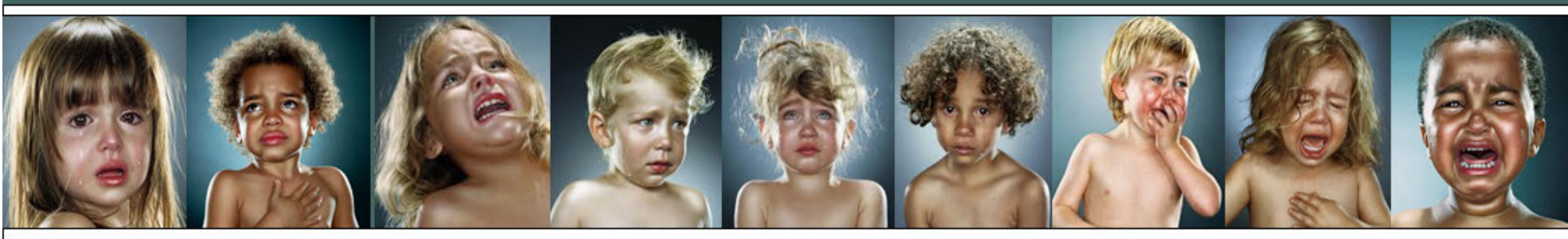

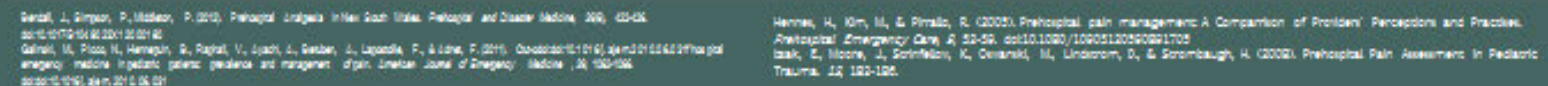




\section{Introduction}

Accurate prehospital assessment of intoxication and associated risks to the patient is crucial in determining the need for ambulance transport to hospital.

Methods
- Literature search using Medline,
CINAHL, Cochrane and Scopus
-Primary focus: prehospital
management of intoxicated patients
-No ethical approval required
-Exclusion factors: $>5$ years old, not
published in English, case reviews,
public health awareness campaigns,
related to specific clinical skills
-Selected articles: prehospital impact
of intoxicated patients (5); triage
tools to assess and refer to detox
facility or ED (4)

\section{References}

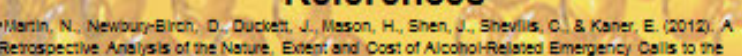

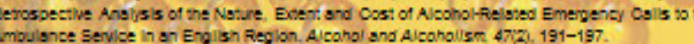

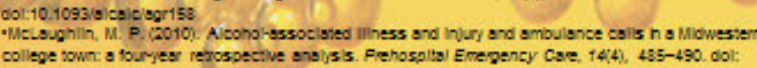

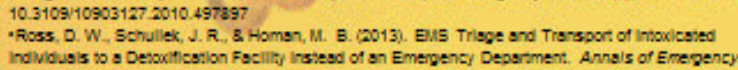

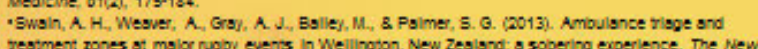

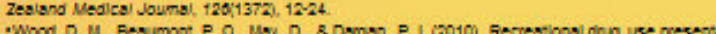

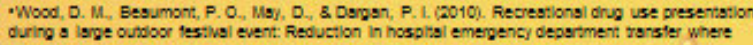

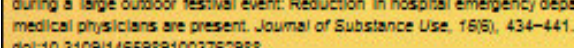

\section{Aim}

To explore issues surrounding prehospital management of intoxicated patients and whether introducing a triage tool would assist St John paramedics in making transport decisions?

reat

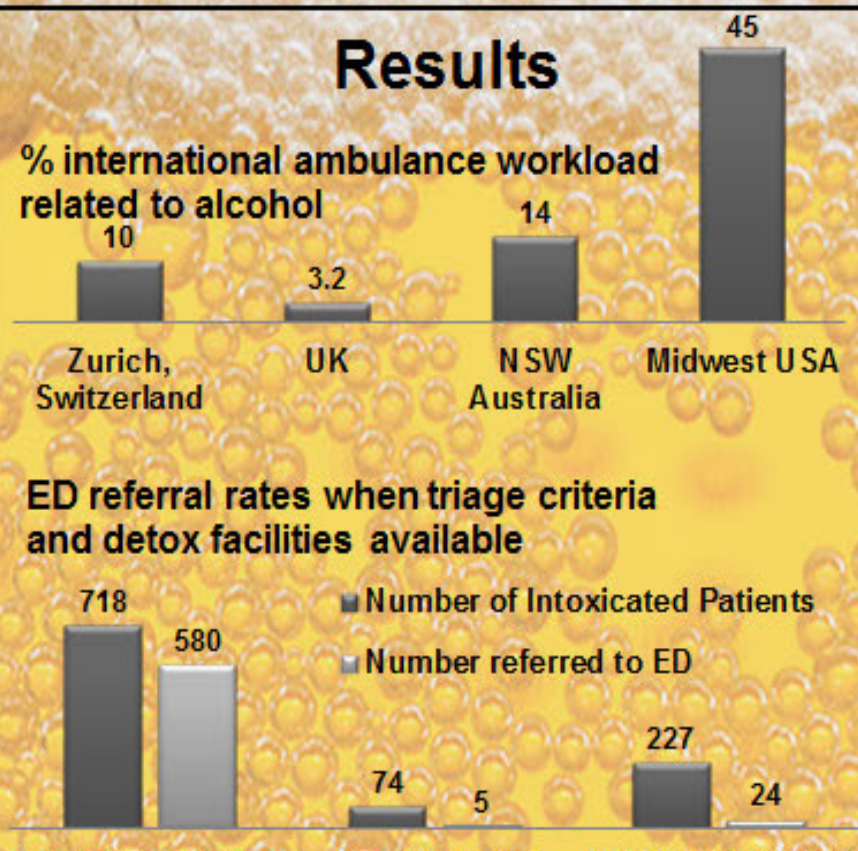

EIPaso EMS 2003. WFA Rugby 7s UK Mus ic Festival 05 2012

\section{Conclusions}

-Introducing an intoxicated-patient assessment tool, utilising the Clinical Help Desk and further training on assessing intoxicated patients would assist St John paramedics in managing this cohort.

-Implementing an alternative care pathway (e.g. 'sobering centre') during peak workload hours may reduce the number of low-acuity alcohol-related ED presentations but requires a multi-agency approach. 


\section{THE POTENTIAL BENEFIT AND FEASIBILITY OF WIDESPREAD INTRODUCTION OF PREHOSPITAL THROMBOLYSIS IN NZ}

\section{INTRODUCTION}

Effective treatment of STEMI patients focuses on minimising time to reperfusion however this is difficult in NZ. Prehospital thrombolysis (PHT) could be effective at reducing treatment times in NZ. This study aims to evaluate the potential benefit and feasibility of widespread introduction of PHT in NZ.

\section{METHODS}

A literature review was conducted by a single independent reviewer. Databases accessed: Cochrane, Medline and CINAHL plus with full text. Key search terms used: paramedic*, ambulance ${ }^{*}$, EMS ${ }^{*}$, prehospital, thrombolysis, New Zealand, AMl and STEMI.

RESULTS OF THE LITERATURE REVIEW EFFECT OF PHT ON MEDIAN CALL TO NEEDLETIMES

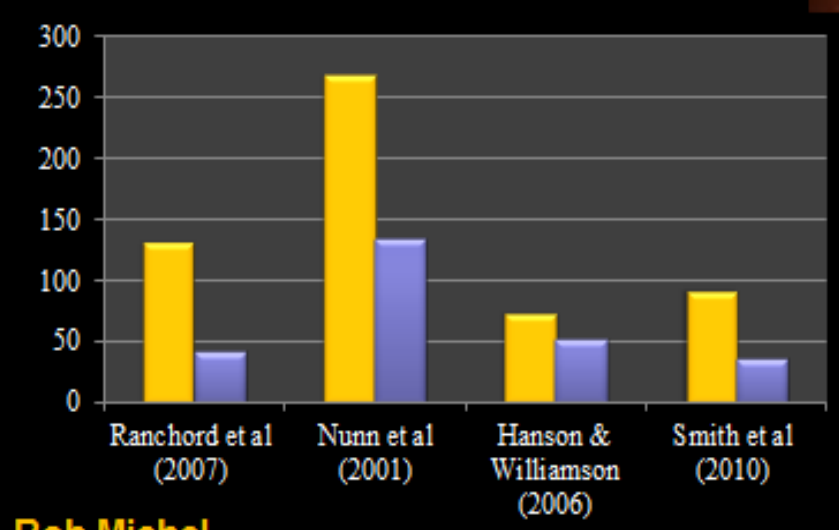

Rob Michel

SN 1062652
- Inhospital Thrombolysis (mins) - Prehospital Thrombolysis (mins)
FACILITATORS OF PHT

- Paramedics generally have a positive attitude towards PHT due to its potential benefit

- Paramedics can accurately interpret 12 lead ECGs and identify eligible patients

- Effective training and structured introduction programmes have lead to successful programmes internationally

\section{BARRIERSTO PHT}

- Difficulties with ECG transmission

- Concerns over some paramedics' ability to thrombolyse safely

- Organisational issues compromising paramedics' willingness to thrombolyse

- Issues regarding thrombolysing whilst single crewed and availability of appropriate resources

\section{CONCLUSION}

There is potential benefit of widespread PHT in NZ. PHT has been successfully introduced internationally and widespread introduction in NZ may be feasible, however further NZ based study is required 


\section{Ambulance Services and End of Life Care;}

\section{Is enough being done and can the service be improved?}

\section{Study Aim:}

To examine the current role of ambulance in end-of-life care.

To examine how this service could be improved.

说 To identify improvement strategies.

\section{Discussion:}

\& Ambulance clinicians assist with end of life patients in a number of ways. They provide this assistance without the benefit of an accurate patient history (Glaetzer, 2011).

- Ambulance clinicians provide assistance without the benefit of a specialist training programme in end of life care (Stone et al., 2009).

- Ambulance clinician involvement may not match patient preferences as there is a lack of communication between allied health professionals and ambulance clinicians (Wiese et al., 2009) and a lack of specialist training for ambulance clinicians (Stone et al., 2009).

- Ambulance clinicians, along with other allied health professionals have a poor understanding of advanced directives (Ruler, 2011) and therefore often resort to resuscitation as a default course of action (California Healthcare Foundation, 2010).

\section{Method:}

Literature Review using only research completed within the past 10 years and only research reports written in English.

Fesearch collected using up-to-date health databases.

层 Research used came from both ambulance studies and allied health professional studies.

The Current Role of Ambulance Clinicians:

† Unplanned Role: Ambulance Clinicians may be called on to assist when health deteriorates and current conditions are not being coped with by onsite carers (AACE,2012).

+ Planned Role: Transfers between care facilities and preferred places of death. (AACE,2012).

Recommendations:

At the Point of Death: May be the first trained professionals on scene right at the

A specialist end-of-life care multi-agency point of death. Ambulance clinicians may developed training programme be offered for all ambulance have to make decisions regatding resuscitation. Ambulance clinicians may provide immediate bereavement support clinicians (AACE,2012).

The development of policies that allow for better information sharing between ambulance personal and other allied services working with patients in the endof-life care pathway ( $A A C E, 2012)$.

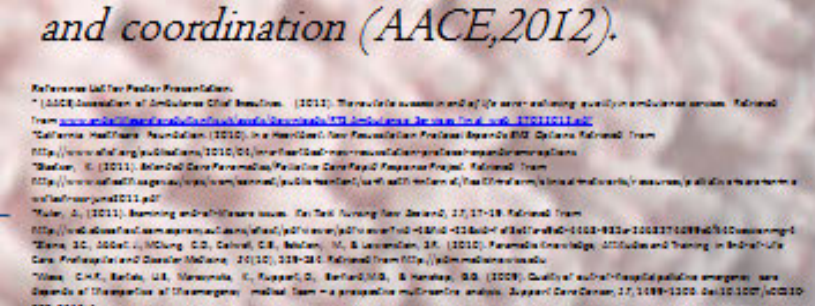




\section{Is Magnesium Sulphate for Eclampsia Indicated in the Pre- Hospital Setting?}

\section{INTRODUCTION}

Preeclampsia is a rare, but potentially life threatening, multisystem disorder of pregnancy, characterized by raised blood pressure and proteinuria, after 20 weeks of gestation. The most dangerous complication of preeclampsia is eclampsia, which is defined by general tonic-clonic convulsions before or after birth.

Eclampsia remains one of the main causes of maternal death.

Up to one third of eclamptic seizures occur out of hospital.

Presently in NZ there are no specific paramedic procedures for eclamptic seizures; midazolam is used for all seizure management. A literature review was undertaken to determine current best practice for the initial management of eclamptic seizures in the pre-hosptial setting. Emergency management of seizures and subsequent safe transfer is essential to minimize morbidity and mortality.

\section{AIM}

To explore the suitability of magnesium sulphate $\left(\mathrm{MgSO}_{4}\right)$ for pre-hospital treatment of eclampsia

\section{METHODS}

A literature search was conducted by a single independent reviewer of English language publications.

Key search terms: eclampsia and preeclampsia

Subheadings: magnesium, treatment, prehospital, emergency and ambulance

Databases used: EBSCO (includes CINAHL and MEDLINE) and SCOPUS

All relevant clinical trials, reviews and procedures from 1995 - 2011 were extracted. Only human studies were considered. The Cochrane database of systematic reviews was searched for reviews of $\mathrm{MgSO}_{4}$ therapy with preeclampsia and eclampsia. Reference lists of identified articles were examined to find additional relevant studies. 62 papers identified; 31 papers provided specific information on preeclampsia and eclampsia

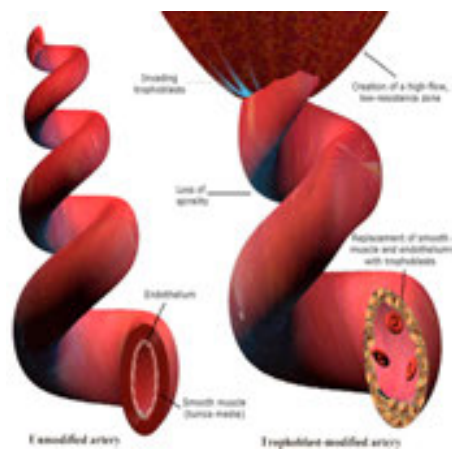

System disturbances with preeclampsia:

Renal: proteinuria, oliguria

Haematological: DIC, hemolysis, thrombocytopenia

Hepatic: epigastric \& RUQ pain

Neurological: seizures, stroke, pulmonary edema, hyperreflexia, placental abruption, severe

headache, visual disturbance, fetal growth retardation

Figure 1: Blood vessel changes with preeclampsia ${ }^{[1]}$

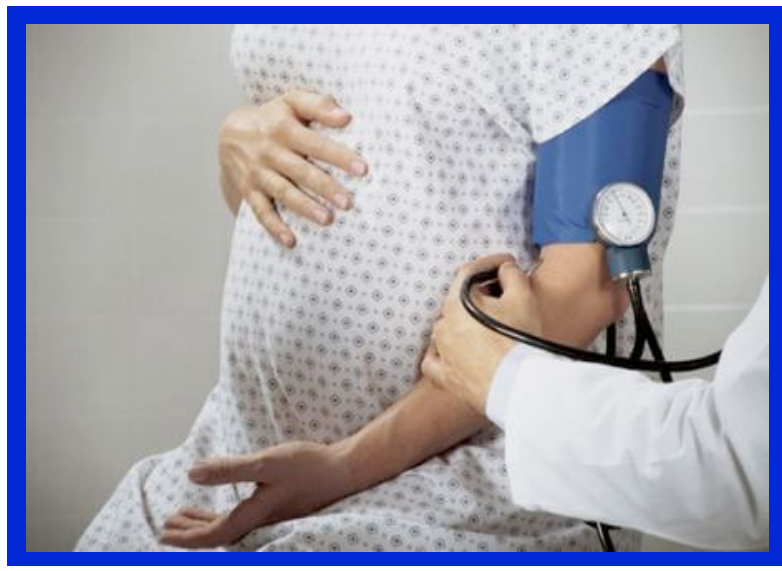

Figure 2: Blood pressure assessment in an at risk patient ${ }^{[2]}$

\section{LITERATURE REVIEW RESULTS}

The literature review extracted nine quality prospective studies, four of which included a control group and three prospective studies which were randomised. Two retrospective reviews were found, one of which included all members of the Society of Obstetricians and Gynaecologists of Canada. Five systematic Cochrane reviews of literature were identified. These five reviews critiqued three to fifteen trials of varying sizes, which the Cochrane reviewers described as generally of good quality. The majority of the fifteen literature reviews were systematic in approach to their review, citing a large number of relevant articles. For example Briggs \& Wan ${ }^{[3]}$ analysed a number of randomised, controlled studies, retrospective studies and observational studies and concluded that $\mathrm{MgSO}_{4}$ was superior to phenytoin and diazepam in the prevention of eclampsia.

The search of grey literature, not found through the conventional channels, identified six sets of practice guidelines, an editorial summary and a seminar proceedings article.

\section{PRACTICAL APPLICATION}

There is international consensus that: $\mathrm{MgSO}_{4}$ is the treatment of choice for eclampsia.

There is good evidence that imminent eclampsia should also be treated prophylactically with $\mathrm{MgSO}_{4}$, to prevent progression to eclampsia.

As eclampsia is a relatively rare condition, regular, updated education and training would be needed.

Recommending changes in paramedic practice will raise issues that require further research and development. This includes but is not limited to: education, cost benefit analysis, and multiuse opportunities for $\mathrm{MgSO}_{4}$

"severe preeclampsia is very high risk, with no room for complacency in its management" ${ }^{41}$ 


\section{Should Paramedics Perform Advanced Cardiac Life}

\section{Support using Automated External Defibrillators?}

Luke SUMMERS

Background: Current NZ paramedic guidelines advocate manual defibrillation over Automated External Defibrillator (AED) by trained users during resuscitation. However, AED mode offers greater accuracy in dysrhythmia identification and timing of rhythm analysis and shock delivery. This study investigates which mode is preferred for delivering shocks during Advanced Cardiac Life Support (ACLS) by paramedics.

Methods: A systematic review of Scopus and EBSCO searched for studies on paramedics performing advanced cardiac life support using both manual and automated external defibrillation modes.

Results: Two studies met the inclusion criteria and were included in the review: i) a prospective observational study of 44 cardiac arrest cases, ii)a manikin study. Both studies supported the use of manual mode.
Discussion: Manual mode reduces interruptions to compressions reducing the duration of pre-shock pauses and increasing the rate of ROSC.

The impact of the defibrillation mode on other components of resuscitation (eg timing of shock delivery, appropriateness of shock, and timing of adjuncts including intubation and drug therapy) requires further research.

Research into paramedic resuscitation is scarce and this study was limited by identification of only a low number of studies.

Conclusion: Paramedics should use manual mode to deliver shocks where possible, if trained to do so. More research is needed to find techniques to reduce no-flow-time when using Lifepak 12 defibrillators in AED mode. 


\section{ANEXPLORATION AND COMPARISON OF CIVILIAN AND MILITARY MANAGEMENT OF TRAUMATIC BRAIN INJURY (TBI)}

\section{The Aim: To identify opportunities to inform practice in the pre-hospital setting and improve patient outcomes.}

Background-TBI cause $>50 \%$ of Adult Trauma Deaths in Civilian Populations. TBl patients in combat (Afghanistan and $(r a q)^{1}$ represent a unique population when compared to civilian populations ${ }^{1}$. TBI in current conflict is described as the "signature injury" 2,3 . The incidences of TBI in living combat causalities $>22 \%{ }^{2}$. $42 \%$ of UK KIA in Afghanistan and Iraq (Apr 06 - Mar 07) had non-survivable $\mathrm{TBI}^{4} .55 \% \mathrm{TBI}$ are associated with injuries to the extremities, which require prevention of catastrophic haemorrhage \& hypotensive resuscitation ${ }^{5}$. The nature of military grade weaponry and its effects resulted in significantly higher rates of $\mathrm{TBI}$ in current conflicts than in World War II, the Korean and Vietnam $\mathrm{War}^{3}$. Previously un-survivable patients are now salvageable due to rapid early intervention and persistent control of secondary injuries ${ }^{6}$.

Objective- To review civilian and military medical articles relating to $\mathrm{TBI}$ and compare the management, outcome and treatment trends.

Methods- Review of 12 civilian and 16 military online journal articles relating to pre-hospital management of TBI.

Search Limitations-

1.English articles 2.Aged $>18$ years. 3.Period 2002-2012. 4.Limit other non matched articles.

Key Findings- Significant differences in,

Mechanism of injury

-Pre and in hospital mortality,

-Number of in-hospital surgical interventions,

- Significance of preventing a single hypotensive episode.

Findings- 1 . There is no treatment for the primary brain injury. The prevention and treatment of the secondary brain injury must be the focus of care. Military TBI is often associated with poly-trauma and its associated hypoxia (arterial oxygen saturation of $<90 \%$ ) and hypotension (systolic blood pressure (SBP) $<90$ $\mathrm{mmHg})^{1}$

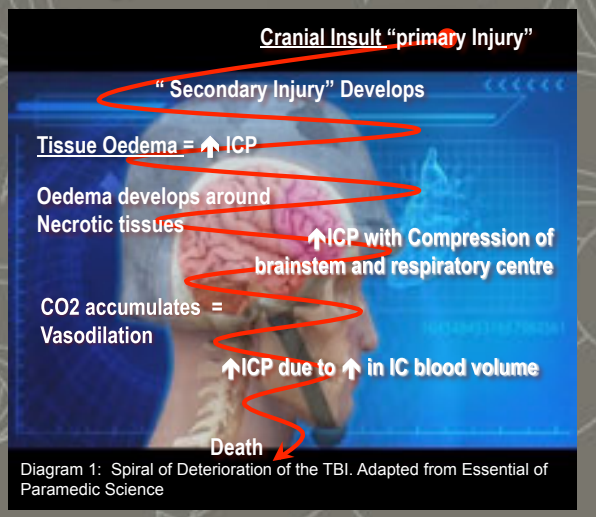

2.Mechanism of Injury (MOI) The morphology of $\mathrm{TBl}$ is complex and difference between civilian and military populations. The insurgent's weapon of choice is the roadside bomb (IED). Military TBI patients $(81.4 \%$ blast \& penetrating injury) Civilian population $(7.2 \% \text { penetrating })^{7}$

This results of an IED, catalogue of four insults:

1. The primary blast,

2. The secondary blast injuries

(i.e. penetrating injuries)

3. Impact injuries from being thrown about and

4. Burns caused by gases \& heat release by explosion

3. The lower rate of in hospital deaths at military facilities compared to civilian facility $(20 \%-25 \%$ vs. $>60 \%)$ tells a different story than would appear.

There is a high rate of death (killed in action) in the military cohort. The profound effects of blast and the significant delays in evacuation remain the most critical events to military combat patients.
Conclusion-

-Prevention of any single hypotensive event is likely to be the single intervention to benefit both military and civilian TBI patients.

- Improved evacuation of military casualties and an increase in surgically invasive management within the civilian sector would see improved mortality of TBI patients.

-Lesson learnt in combat support a more aggressive approach to nohypotensive resus in standalone TBI and places the emphasis of care on effective assessment, utilising tools like GCS and pupil evaluation, a clear area for excellence in paramedic practice.

-Literature supports current treatment guidelines, the emphasis on preventing hypotension and hypoxia should be reinforced. The MOI in combat results in higher death from wounds but aggressive in hospital care is resulting in better outcomes for TBI patients.

-Although there is a long-standing tradition of advancement in cares gained from experience in combat by military medics, it would appear that in the prehospital arena on this occasion, is not be the case.

-The austere environment of the military medic and the profound effect, of the methods of operation, by insurgents, provides little evidence of improving the prehospital outcome for military TBI patients

The Numbers Game

$50 \%$ Percent of Adult Trauma deaths from TB

$>22 \%$ Percent incident of TBI in live Combat patients

$42 \%$ Percent of TBI resulting in non-preventable deaths

$5 \% \mathrm{TBI}$ associated with other injuries

81.4\% Military TBI from Blast, Penetrating injuries

$7.2 \%$ Civilian TBI from Penetrating Injuries

$>16 \%$ TBI patients arriving at hospital hypotensive

$50 \%$ Number TBI patients who have had a hypoxic event

$50 \%$ Of mil TBI die immediately or shortly after injury

$25 \%$ In Hospital Death rate of Military TB

$>60 \%$ In Hospital Death rate of Civilian TBI

$3.3 \%$ Mortality of Died of Wounds (DOW) Vietnam

$5.3 \%$ Mortality of Died of Wounds Iraq \& Afghanistan

$21.1 \%$ Total Mortality (DOW + KIA) Vietnam

$16.1 \%$ Total Mortality (DOW + KIS) Iraq \& Afghanistan

45min Average evac time (1968-69) Vietnam

$1 \mathrm{hr} 49 \mathrm{~m}$ Average evac time lraq 2005 (UK Forces)

7hrs Average evac time Afghanistan 2004 (UK Forces)

Brendan Wood 0818118 